\title{
X-BAND BACKSCATTER MAP GENERATION USING TERRASAR-X DATA
}

\author{
Paola Rizzoli, Benjamin Bräutigam, Steffen Wollstadt, Josef Mittermayer \\ German Aerospace Center (DLR), Germany
}

\begin{abstract}
The goal of this work is the generation of an X-Band backscatter map by assembling images acquired by the TerraSAR-X mission. Global backscatter data is required for accurate performance estimation and instrument commanding inside the TerraSAR-X and TanDEM-X missions. Moreover, many scientific applications can be based on the analysis of backscatter behavior and evolution. The complete ground coverage will be achievable with TanDEM-X mission data. An interpolator, that allows the estimation of the backscatter for any required polarization and incidence angle from the available data, has been implemented. In this paper, the backscatter map generation algorithm will be presented, together with the first obtained results, generated using TerraSAR-X data. Moreover, the validity of the interpolation models will also be discussed, presenting the preliminary results of a statistical analysis of backscatter from TerraSAR-X data.
\end{abstract}

Index Terms - X-Band Backscatter, TerraSAR-X.

\section{INTRODUCTION}

TerraSAR-X (TSX) and its twin satellite TanDEM-X (TDX) are two new German SAR satellites, developed in a public/private partnership between DLR and EADS Astrium [1]. The main target of the TanDEM-X mission is the generation of a worldwide, high precision Digital Elevation Model (DEM), according to the HRTI-3 specification [2]. To do so, both satellites will fly in close orbit configuration, enabling the acquisition of highly accurate cross- and along-track interferograms. A high accuracy in commanding and performance is needed, in order to fulfill such high-demanding tasks. For both TerraSAR-X and TanDEM-X missions, precise X-Band backscatter data is necessary for an optimized operation of the SAR system. For known backscatter characteristics of a requested SAR scene, the commanded receive gain can be adapted to mitigate clipping or signal saturation. For realistic performance prediction of the SAR and DEM products, the backscatter information is a highly valuable input (e.g. for SNR estimation and height error calculation). Moreover, the availability of new X-Band backscatter maps, generated using TSX and, later on, also TDX data, leads to many future scientific applications, such as the monitoring of backscatter evolution in time and the study of reflectivity behavior depending on the atmospheric conditions. Sections 2 and 3 show the map generation algorithm and a preliminary validation using a statistical approach. Section 4 presents the interpolator of missing values, while the first results obtained for the generation of a global and on-demand X-Band backscatter maps are finally displayed in section 5 .

\section{BACKSCATTER MAP GENERATION}

The generation of a backscatter mosaic requires two different steps: a dedicated processing for each input image, in order to retrieve a matrix of absolutely calibrated radar brightness values $\beta^{0}$, and the assembling of the suitable data inside the desired ground region, referred to the required incidence angle. Since the total high amount of data to deal with requires the optimization of the processing time and, moreover, high resolution is not needed, TSX quicklook images have been considered as input data [3]. Such images provide less resolution regarding the standard SAR image, by a factor that depends on the acquisition mode. The default resolution for the output global backscatter map has been chosen to $5 \times 5 \mathrm{~km}^{2}$ at the equator. For such a reason, the input quicklook images are first averaged by a factor depending on the acquisition mode, in order to reduce the total amount of data that has to be stored to images of similar resolution. Each input image is then geocoded and, for every pixel, the correspondent incidence angle is evaluated, leading to the generation of an incidence matrix associated to each product. Each image has to be absolutely calibrated, in order to convert a single pixel digital number $D N$ into the correspondent radar brightness $\beta^{0}$ in the following way:

$$
\beta^{0}=D N^{2} / K
$$

where $K$ represents the calibration parameter, obtained from the combination of the processing gain and the specific calibration constant. Notice that it is possible to retrieve the backscatter coefficient $\sigma^{0}$ directly from $\beta^{0}$, by compensating the dependence from the local incidence angle $\alpha$ :

$$
\sigma^{0}=\beta^{0} \sin (\alpha)
$$

At this point, every single processed product is stored inside a long term data base, together with some additional parameters connected to it, such as the incidence angles matrix, 
the geocoded coordinates matrix, the acquisition times and the polarization channel. All these additional parameters are available later on to generate on-demand backscatter maps, for example using data acquired only inside a certain temporal interval or seasonal period, as described in section 5 .

Figure 1 presents the algorithm flowchart for the assembling process of calibrated data into a final mosaic. It is well known that, for a given pixel, the amplitude of the radar backscatter depends on several parameters, such as the incidence angle, the surface type, the polarization and the radar frequency. All this factors must be taken into account during the generation process.

Once the desired region of interest and other input parameters, such as polarization, reference incidence angle (explained below) and acquisition interval, have been properly set by the user, the $\beta^{0}$ database is accessed, to retrieve a list of all the available calibrated data for the required output map. Since the backscatter amplitude changes depending on the ground characteristics (e.g. type of vegetation, ice-covered regions) and on the incidence angle, it has been decided to refer the output backscatter map to a precise reference incidence angle $\alpha_{\text {ref }}$, instead of simply applying the angular compensation described in equation 2. For this reason, a dedicated interpolator has been implemented, able to convert $\beta^{0}$ values, acquired with different incidence angles, to the reference $\alpha_{r e f}$. The first step consists in associating to each pixel a proper vegetation class. A classification map provided by esa, with on-ground resolution of $300 \times 300 \mathrm{~m}^{2}$, has been used [4]. The $\mathrm{X}$-Band backscatter models retrieved from [5] have been used to implement the interpolator. As an example, Figure 2(a) shows the averaged pixel intensity versus the incidence angle for different vegetation classes and $\mathrm{HH}$ polarization. The following function has been used to interpolate the backscatter models:

$$
\sigma_{0}=c_{0}+c_{1} \exp \left(-c_{2} \alpha\right)+c_{3} \cos \left(c_{4} \alpha+c_{5}\right)
$$

where $c_{0}, \cdots, c_{5}$, represent the $\sigma^{0}$ fitting coefficients and $\alpha$ is the incidence angle.

For each considered vegetation class, a correction curve has been computed, by inverting such models. By applying the proper correction value to each image pixel, depending on the acquisition incidence angle, we are now able to generate a mosaic of $\sigma^{0}$ values which is completely referred to a single incidence angle value $\alpha_{\text {ref }}$ (Figure 2 (b)). Notice that more than one input value can belong to the same output cell. For this reason a proper way to average multiple pixels inside the same cell has been found and three different maps are generated as outputs, respectively representing the mean value, the maximum and the standard deviation of all the available pixels inside a single output resolution cell.

Figure 3 shows an example of a backscatter map over Australia for $\mathrm{HH}$ polarization, with a reference incidence angle of 25 [deg] and a sampling step of 0.05 [deg] in lat/lon coordinates. Notice that not all the required data have been

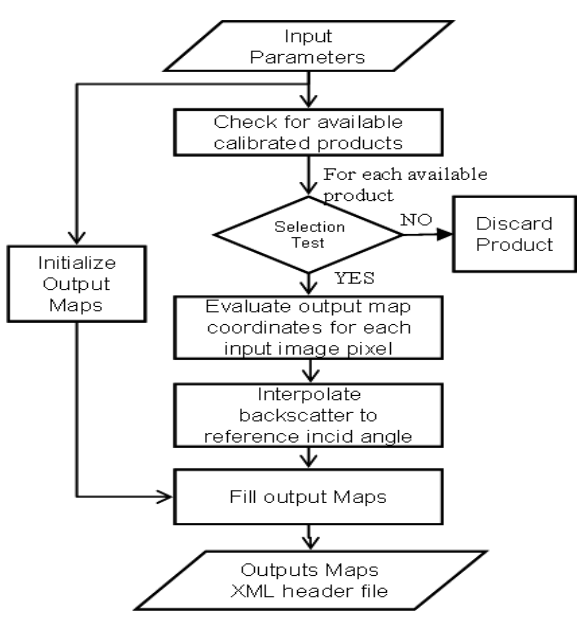

Fig. 1. Mosaic Generation flowchart.

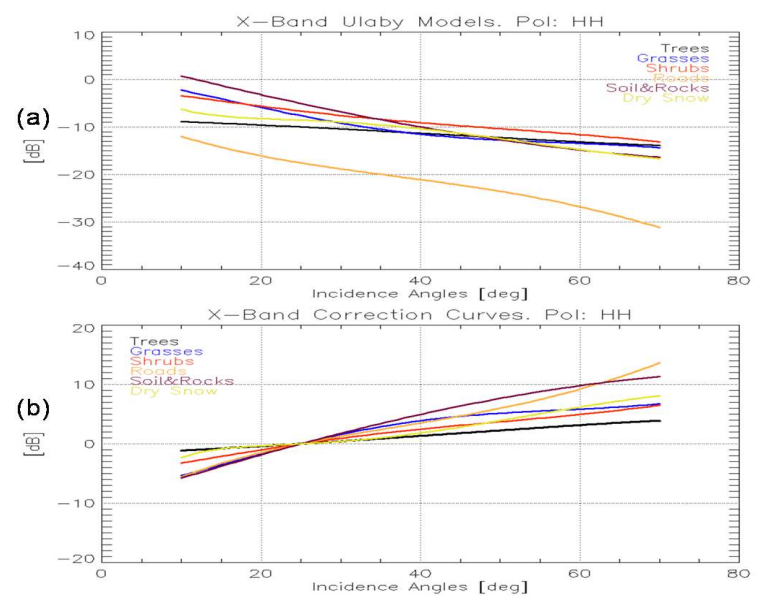

Fig. 2. (a) - X-Band backscatter dependency with the incidence angle. Ulaby Models (mean value). (b) - Correction curves referred to $\alpha_{\text {ref }}=25[\mathrm{deg}]$.

acquired yet, so that some missing values are still present, as it can be inferred from the data classification mask in figure 3(d).

\section{STATISTIC ANALYSIS OF BACKSCATTER BEHAVIOR}

The backscatter map interpolator was implemented taking into account the reflectivity models proposed by F. T. Ulaby in [5]. In order to provide a validation of such models and of the interpolation process itself, a statistical analysis of backscatter reflectivity for TSX data is currently being performed. Backscatter values coming from different products are analyzed, grouping them together depending on the polarization channel and the vegetation class. For a limited number of incidence angles intervals, the histogram of backscatter can be evaluated. A gaussian fitting of the distribution is 

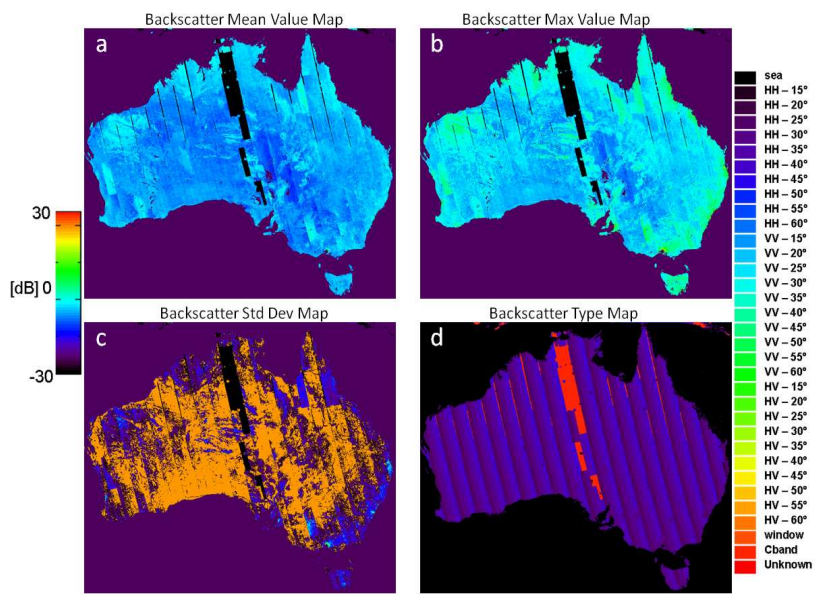

Fig. 3. Backscatter map example over Australia, HH pol, 25 [deg] incidence. (a) mean value. (b) max value. (c) Standard deviation. (d) Data classification mask.

then performed, and the retrieved mean values can be used to implement a non linear weighted fitting along the incidence angles dimension, as explained in figure 4 . In this way, a model of backscatter behavior, depending on vegetation type and incidence angles, can be retrieved and compared to the models in [5]. A further purpose of this analysis resides also in the future generation of a complete database of backscatter models for X-Band, generated using spaceborne data only.

\section{FILLING DATA GAPS}

Since the global earth coverage of TSX data is not available yet, some ground regions remain uncovered. In order to provide a complete map, required i.e. for commanding applications, such areas must be filled. The idea is to use TSX data acquired using different polarizations first and interpolate them for the right polarization and incidence angle in the same way as explained in section 2 . Then, small gaps can be filled by averaging the nearest available samples. Finally, if empty areas are still present, they can be filled by a coarse CBand backscatter map, referred to X-Band. Figure 5 presents the algorithm flowchart, while Figure 6 shows an example of the interpolated backscatter mean value of Australia (left) and its correspondent classification mask (right).

\section{X-BAND BACKSCATTER MOSAICS}

In the current section the preliminary results obtained for the generation of global and on-demand X-Band backscatter maps are presented.

Figure 7 shows the backscatter mean value, generated using all the available TSX data, acquired from October 2008 until May 2010, for $\mathrm{HH}$ polarization and a pixel spacing of 0.05 [deg] in lat/lon coordinates (which corresponds to an on-

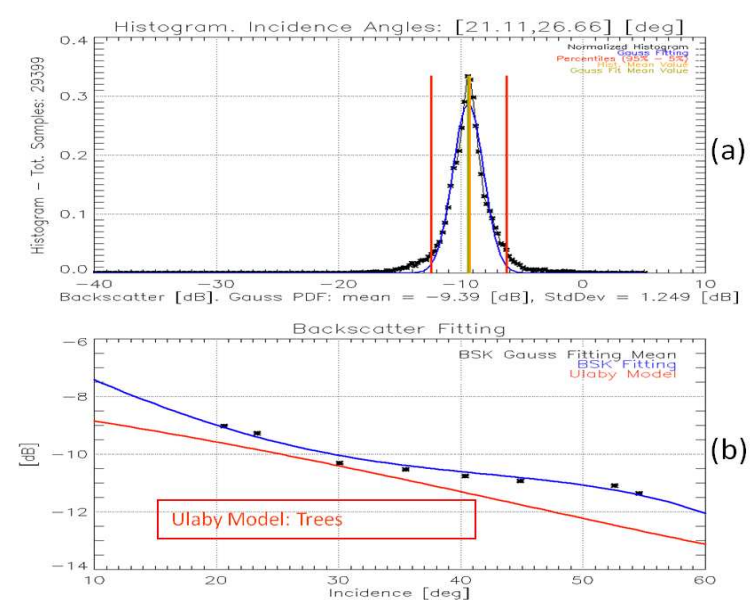

Fig. 4. (a) - Backscatter histogram and gaussian fitting for the vegetation class of mixed broad-leaved and needle-leaved forest, for incidence angles inside the [21.5 deg, $26.5 \mathrm{deg}$ ] interval. (b) - Weighted non linear fitting of gaussian mean values for different incidence angles (blue). Ulaby model for the correspondent vegetation class (red).

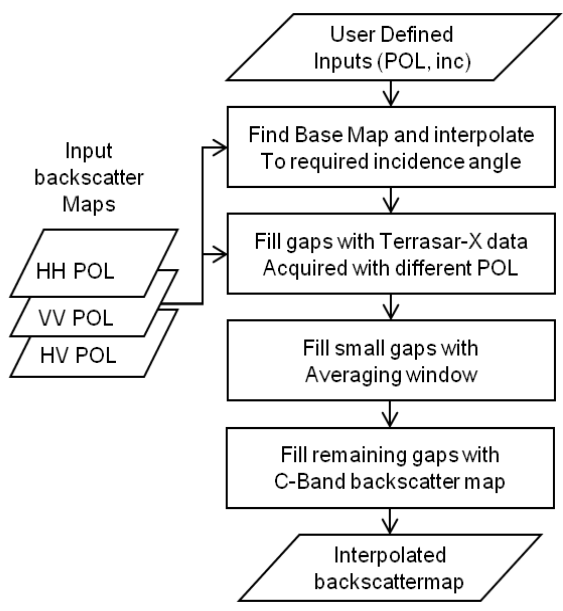

Fig. 5. Filling data gaps algorithm flowchart.

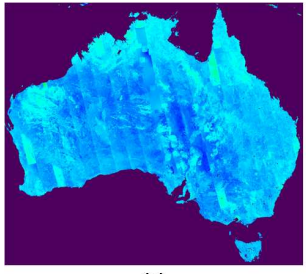

(a)

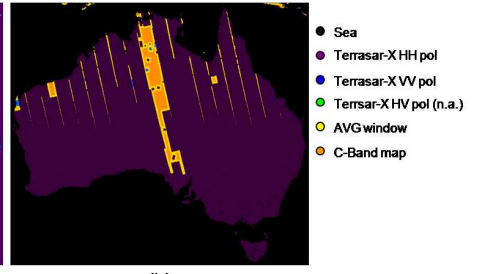

(b)
Fig. 6. Backscatter map example over Australia after missing values interpolation, $\mathrm{HH}$ pol, 25 [deg] incidence. (a) mean value. (b) Classification mask.

ground resolution of about $5 \times 5 \mathrm{~km}^{2}$ at the equator). Figure 8 presents the global backscatter map after the application of 
the interpolation processing for missing values.

Moreover, the algorithm flexibility allows the generation of on demand backscatter maps, that can be useful for different scientific purposes. For example, the user can define several parameters, such as the desired output resolution, the satellite orbit direction or the seasonal time of the input data. In this this way, the backscatter dependency on the sensor parameters or its evolution depending on time and weather conditions can be monitored. Figure 9 presents a backscatter mosaic of Iceland with a ground resolution of $200 \times 200\left[\mathrm{~m}^{2}\right]$, generated using 7 scanSAR images, acquired in $\mathrm{HH}$ polarization and ascending orbit. All data were acquired from December 2008 to February 2009, during the winter period.

\section{SUMMARY}

In this paper, the actual work developed for the generation of $\mathrm{X}$-Band backscatter maps using TerraSAR-X data has been discussed. The processing approach and the first results obtained for the generation of a global backscatter map have been presented. Moreover, on-demand backscatter maps, characterized by higher resolution, can be generated as well. Not enough data have been acquired with TerraSAR-X satellite yet, in order to generate a complete global backscatter map, covering the whole earth. The full coverage will be achieved with the systematic acquisitions provided during the TanDEM-X mission.

The importance of having a complete global backscatter map resides in the many further applications that require its use, such as instrument commanding and performance estimation. For this reason an interpolator has been implemented, filling gaps with corrected data from other polarizations, neighbor samples or even other sensors. The validation of the entire process is under testing and a statistical analysis of the $\mathrm{X}$-Band backscatter behavior is being performed.

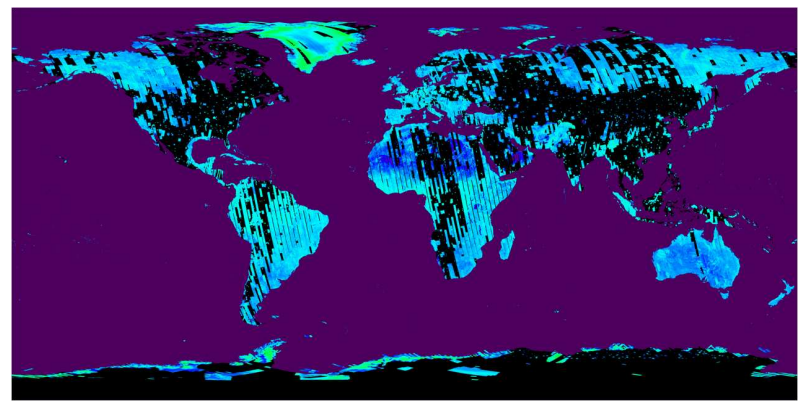

Fig. 7. X-Band global backscatter map (mean value) for $\mathrm{HH}$ polarization, generated using all the TSX available quicklook data, acquired from October 2008. Values are saturated inside the interval of $[-30,+30] \mathrm{dB}$.

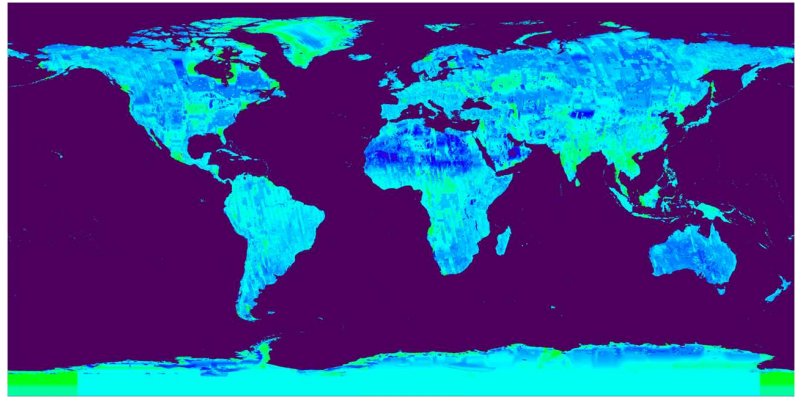

Fig. 8. X-Band global backscatter map (mean value) for $\mathrm{HH}$ polarization, after filling gaps of missing data.

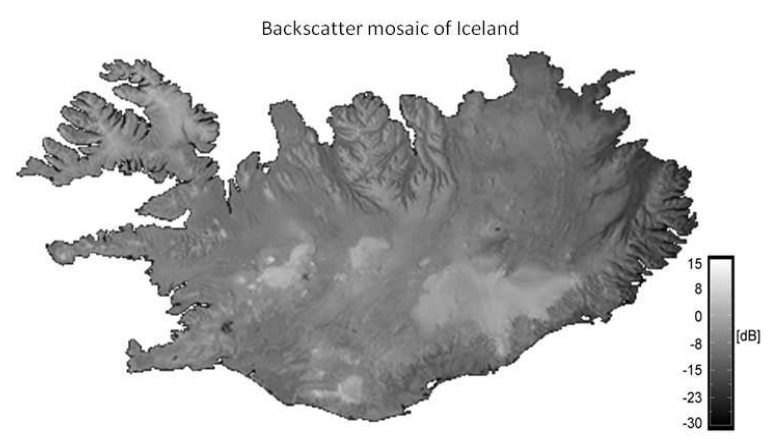

Fig. 9. Mosaic of 7 scanSAR images acquired from December 2008 to February 2009. Polarization: HH, incidence: 30 [deg], resolution 200x200 [ $\left.\mathrm{m}^{2}\right]$, orbit direction: Ascending, season: Winter.

\section{REFERENCES}

[1] Buckreuss S.; Werninghaus R.; Pitz W.: German Satellite Mission TerraSAR-X, 2008 IEEE Conference, Rome, Italy, 2008

[2] Krieger G.; Moreira A.; Fiedler H.; Hajnsek I.; Werner M.; Younis M.; Zink M.: TanDEM-X: A Satellite Formation for High-Resolution SAR Interferometry, IEEE Transactions on Geos. and Remote Sens., vol. 45, no. 11, pp. 3317-3341, Nov. 2007

[3] Fritz T.; Eineder M.; Breit H.; Schättler B.; Boerner E.; Huber M.: The TerraSAR-X Basic Products - Format and Expected Performance, Eusar Proceedings, 2006

[4] GLOBCOVER Products Description Manual, http://ionial.esrin.esa.int/images/ GLOBCOVER_Product_Specification_v2 . pdf

[5] Ulaby F. T.; Dobson M. C.: Handbook of Radar Scattering Statistics for Terrain, Norwood, MA: Artech House, 1989 